\title{
Pelvic pain correlates with peritoneal macrophage abundance not endometriosis
}

\author{
Douglas A Gibson', Frances Collins', Bianca De Leo 1,2, Andrew W Horne² and Philippa T K Saunders' \\ ${ }^{1}$ Centre for Inflammation Research, University of Edinburgh, Edinburgh, UK \\ 2MRC Centre for Reproductive Health, Queen's Medical Research Institute, University of Edinburgh, Edinburgh, UK
}

Correspondence should be addressed to D A Gibson: D.A.Gibson@ed.ac.uk

\begin{abstract}
Endometriosis is a chronic neuroinflammatory pain condition affecting $\sim 180$ million women worldwide. Surgical removal or hormonal suppression of endometriosis lesions only relieves pain symptoms in some women and symptomatic relapse following treatment is common. Identifying factors that contribute to pain is key to developing new therapies. We collected peritoneal fluid samples and clinical data from a cohort of women receiving diagnostic laparoscopy for suspected endometriosis $(n=52)$. Peritoneal fluid immune cells were analysed by flow cytometry and data compared with pain scores determined using the pain domain of the Endometriosis Health Profile Questionnaire (EHP-30) in order to investigate the association between peritoneal immune cells and pain symptoms. Pain scores were not different between women with or without endometriosis, nor did they differ according to disease stage; consistent with a poor association between disease presentation and pain symptoms. However, linear regression and correlation analysis demonstrated that peritoneal macrophage abundance correlated with the severity of pelvic pain. CD14high peritoneal macrophages negatively correlated with pain scores whereas CD14low peritoneal macrophages were positively correlated, independent of diagnostic outcome at laparoscopy. Stratification by pain subtype, rather than endometriosis diagnosis, resulted in the most robust correlation between pain and macrophage adundance. Pain score strongly correlated with CD14high $(P=0.007)$ and $C D 14$ low $(P=0.008)$ macrophages in patients with non-menstrual pain and also in patients who reported dysmennorhea (CD14high $P=0.021, C D 14^{\text {low }} P=0.019$ ) or dysparunia (CD14high $\left.P=0.027, \operatorname{CD} 14^{\text {low }} P=0.031\right)$. These results provide new insight into the association between peritoneal macrophages and pelvic pain which may aid the identification of future therapeutic targets.
\end{abstract}

\section{Lay summary}

Endometriosis is a common condition where cells similar to those that line the womb are found elsewhere in the body. It is associated with inflammation and pain in the pelvis and affects $\sim 180$ million women worldwide. Current treatments are not effective for all patients and we, therefore, need to understand what causes pain in order to develop new treatments. We investigated the types of immune cells present within the pelvis of women undergoing investigation for suspected endometriosis. Disease diagnosis and stage (I-IV) was recorded along with pain score determined by questionnaire. We characterised the immune cells present and compared them to disease stage and pain score. We found that pelvic pain was linked to the abundance of immune cells but, surprisingly, not to disease stage. These findings suggest that immune cells are closely associated with pain severity in endometriosis and may be good targets for future endometriosis treatments.

Key Words: - endometriosis $\quad$ macrophage $\quad$ peritoneal $\quad$ pain 


\section{Introduction}

Endometriosis is a chronic neuroinflammatory condition affecting 180 million women worldwide and has a socioeconomicimpact similar to diabetes (Horne \& Saunders 2019). It is defined by the presence of endometrial-like tissue outside the uterus ('lesions'), commonly within the pelvic cavity. The most common symptoms of endometriosis are chronic pelvic pain, dyspareunia, dysmenorrhoea and non-menstrual pain (Horne \& Saunders 2019, Zondervan et al. 2020). While the exact aetiology of endometriosis is unknown, changes in inflammatory processes are thought to contribute to the pathogenesis of the disease.

The definitive method to diagnose endometriosis is by visualisation at the time of surgery (laparoscopy). Endometriosis is further staged according to the revised scoring system of the American Society for Reproductive Medicine (rASRM) to determine the endometriosis stage based on macroscopic features of the disease including location/appearance of lesions and extent of disease (ranging from I to IV) (1997). Although this staging is commonly used to define disease burden, comparisons between patient-reported severity of pain symptoms and rASRM stage consistently report a poor correlation (Vercellini et al. 2007). For example, a multivariate analysis of over 1000 patients assessing the correlation between endometriosis stage, lesion type and pain severity demonstrated that deep endometriosis is associated with pain symptoms characteristic of anatomical location (dyspareunia) but evidence for an association of other pain subtypes (dysmenorrhea, non-menstrual pain) with disease severity/location was marginal (Vercellini et al. 2007). Dysmenorrhea is reported to be more frequent and to result in higher pain scores in women with endometriosis but this is also independent of lesion location (Fauconnier \& Chapron 2005). Notably, pelvic pain symptoms are common, with up to $90 \%$ of women reporting having experienced dysmenorrhea, $42 \%$ dyspareunia and 39\% acyclic (non-menstrual) pain (Jamieson \& Steege 1996) at some time in their lives. The relationship between pain symptoms and diagnosed endometriosis is further complicated by the identification of lesions in asymptomatic women (Fauconnier \& Chapron 2005). These data demonstrate that identifying potential pathophysiological mechanisms responsible for pain, beyond those associated with endometriosis lesions, remains important as a step towards improved patientfocused therapies.

Peritoneal macrophages are the most abundant immune cell in the peritoneal cavity (Kubicka et al.
1996) with defined roles in clearance of apoptotic cells and immune surveillance. Analysis of peritoneal fluid from women with endometriosis suggests that the inflammatory microenvironment is altered in the peritoneal cavity of women with disease. Notably, increased concentrations of macrophage growth factors and chemokines such as colony-stimulating factor 1 (CSF1) and monocyte chemoattractant protein 1 (CCL2) are detected in peritoneal fluid from women with endometriosis (Arici et al. 1997, Budrys et al. 2012, Beste et al. 2014) and this is associated with increased numbers of peritoneal macrophages compared to women without disease (Hill et al. 1988, Beste et al. 2014, Gogacz et al. 2017). Furthermore, peritoneal macrophage function appears to be dysregulated in endometriosis patients and this is characterised by increased secretion of proinflammatory cytokines and reduced phagocytic capacity (Chuang et al. 2009, Punnonen et al. 1996, Richter et al. 2005, Beste et al. 2014). Peritoneal macrophage dysfunction is thought to contribute to the establishment of lesions in women with endometriosis, although the mechanistic evidence for this is largely based on studies in mouse models. These studies demonstrate that peritoneal macrophages are increased in experimentally induced endometriosis and are required for the vascularisation and growth of endometriosis lesions (Bacci et al. 2009, Capobianco et al. 2011).

Macrophage dysfunction may also affect pain symptoms via secretion of neurotrophic factors that promote nerve growth (Kajitani et al. 2013). Notably, CD68-positive macrophages are reported to co-localise with nerve fibres within peritoneal endometriotic lesions and it has been speculated that they can promote endometriosis pain symptoms in women (Tran et al. 2009). In a mouse model of endometriosis, peritoneal macrophages appeared to promote endometriosisassociated hyperalgesia, putatively via secretion of macrophage-derived insulin-like growth factor 1 (Forster et al. 2019).

Macrophages are highly diverse and we need a better understanding of the different subpopulations present in endometriosis in order to determine if they have distinct functional roles. Studies in other contexts have reported there is heterogeneity within human peritoneal macrophage populations and at least two subsets can be identified by assessing the expression of canonical markers, such as CD14, CD16 and HLA-DR (Ruiz-Alcaraz et al. 2016, 2018) or complement receptor of the immunoglobulin superfamily (CRIg) (Irvine et al. 2016). Notably, these peritoneal macrophage subsets 
appear to be altered in disease states, such as liver cirrhosis (Irvine et al. 2016).

The objective of this study was to phenotype and quantify peritoneal macrophage subpopulations in women undergoing diagnostic laparoscopy for suspected endometriosis and to determine whether this was associated with the presence of endometriosis and/or pain symptoms.

\section{Materials and methods}

\section{Study approval}

Written informed consent was obtained from all study participants prior to surgery; ethical approval was granted by the Lothian Research Ethics Committee (LREC 11/ $\mathrm{AL} / 0376)$. Methods were carried out in accordance with NHS Lothian Tissue Governance guidelines and EPHect guidelines (https://endometriosisfoundation.org/ ephect/).

\section{Clinical samples and patient data}

Eligible participants were women with chronic pelvic pain (aged 18-50 years) of $>3$ months duration who were undergoing diagnostic laparoscopy for suspected endometriosis in NHS Lothian, UK. Pelvic pain was defined as pain located within the true pelvis (between and below the anterior iliac crests). Screening commenced on 23/10/2014 and last participant recruited on $03 / 05 / 2016$. Of the 202 women who were approached for participation, 121 consented to take part. Peritoneal fluid was recovered from 74 women during surgery, of which 52 had sufficient volume for downstream analysis. 22 participants were excluded from the study due to insufficient peritoneal fluid volume. Pain scores were determined using the pain domain of the Endometriosis Health Profile Questionnaire (EHP-30) (a validated endometriosis-specific pain questionnaire completed pre-operatively (20)). Age, BMI, menstrual cycle stage and hormone status were obtained and recorded along with other key clinical data (Supplementary Tables 1 and 2 , see section on supplementary materials given at the end of this article). For 29 women who were not on hormones and had regular menstrual cycles, the diagnosis of endometriosis was confirmed macroscopically at laparoscopy in 19 women, whereas no evidence of endometriosis was found in ten women ('no endo'). Women with endometriosis diagnosis were subsequently classified according to the revised scoring system of the American Society for Reproductive Medicine (rASRM) as stage I $(n=10)$, II $(n=4)$ and III/IV $(n=5)$. For some analyses, the remaining 25 women who were receiving hormone treatment were included. In women who met this criterion, diagnosis of endometriosis was confirmed in 14 women, while 11 were classified as 'no endo' (no obvious pelvic pathology at laparoscopy).

\section{Flow cytometry}

Immune cells recovered from peritoneal fluid were analysed by flow cytometry using standard methods (McKinnon 2018). Briefly, cells were washed and subjected to red cell lysis before being counted and resuspended in FACS buffer at a concentration of $0.5-1 \times 10^{6}$ cells $/ 100$ $\mu \mathrm{L}$. Antibodies were used at manufacturer's recommended concentration (Supplementary Table 3). Cells were gated to select immune cells (live, $\mathrm{CD} 45^{+}$) and further sub-gated to select myeloid cells $\left(\mathrm{CD} 11 \mathrm{~b}^{+}\right)$and exclude $\mathrm{T}, \mathrm{B}$ and NK cells (CD3-, CD19-, CD56-). Gating of subpopulations was performed based on fluorescence minus one (FMO) and population distribution (CD14). This population gating was applied to all samples. Samples were assessed using a Becton Dickinson FACS Aria II. Data were analysed using FlowJo ${ }^{\text {TM }}$ Software.

\section{Statistical analysis}

Statistical analysis was performed using Graphpad prism. $\mathrm{T}$ test was used to test the difference in the means of two groups. Two-way ANOVA was used to determine the significance between treatments in grouped data. Nonparametric testing was utilised where sample sizes were insufficient to confirm normality of data distribution; Kruskal-Wallis test was used to assess differences between multiple groups or Mann-Whitney test to assess variance between two groups. Pearson correlation was used to quantify the degree to which two variables (macrophages abundance and pain score) were related. Criterion for significance was $P<0.05$. All data are presented as mean \pm s.E.M.

\section{Results}

Pelvic pain does not correlate with endometriosis diagnosis or disease stage

Baseline characteristics of the patient cohort were assessed to identify potential factors which may influence pelvic

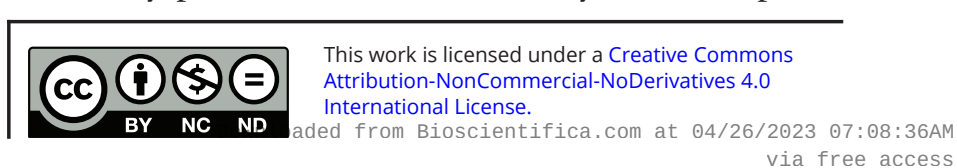


pain in women. Pain scores were determined using the pain domain of the EHP-30 questionnaire (Jones et al. 2001). For women who were not on hormones; Age, BMI, and pain scores did not differ between those women categorised as 'no endo' or those with surgically diagnosed endometriosis (Fig. 1A and Supplementary Table 1). There was no statistically significant difference in pain score between endometriosis rASRM stages I, II or III/IV, although pain scores were highly variable (Fig. 1B). Oral contraceptives and treatments which suppress hormone production are reported to decrease endometriosisassociated pain in some women (Taylor et al. 2017, Crosignani et al. 2006). In this study, pain scores in women receiving hormonal treatment $(\mathrm{H})$ were not different from those women who were not on hormones $(\mathrm{NH})$ and this was true in women with (Fig. 1C) and without (Fig. 1D) a confirmed surgical diagnosis of endometriosis.

Two populations of peritoneal macrophages are present in the peritoneal fluid of women with suspected endometriosis which are not altered by hormone status

Cells were recovered from peritoneal fluid and analysed by flow cytometry. Cells were gated to select immune cells and further sub-gated to select myeloid cells and exclude T, B and NK cells (Fig. 2A and B). Two populations of
A

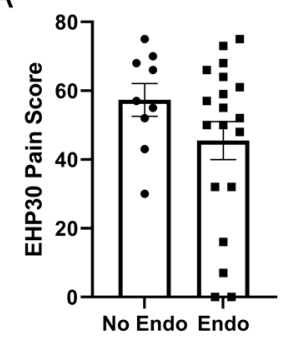

C

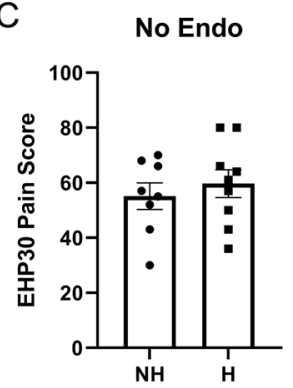

B
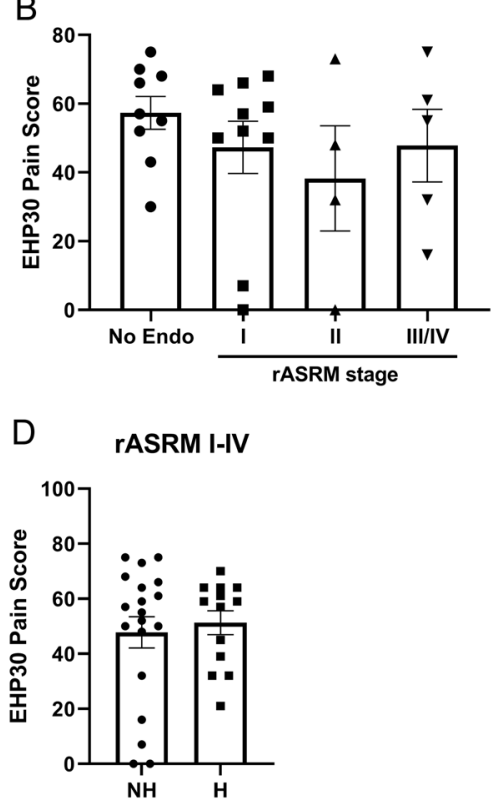

D RASRM I-IV

Figure 1 Pain score does not differ by endometriosis diagnosis, stage of disease or hormone treatment. (A) Presence/absence of endometriosis did not affect the pain score (pain domain of EHP30) and (B) did not differ according to rASRM stage. Hormone treatment did affect pain score in women without (C) or with endometriosis diagnosis (D). peritoneal macrophages $(\mathrm{PM} \varphi)$ were identified which were HLA-DR-positive and had either high or low-intensity expression of CD14 (Fig. 2C).

In women with regular menstrual cycles, the phase of the menstrual cycle (categorised as proliferative or secretory), had no significant impact on the abundance of $\mathrm{CD} 14^{\text {high }}$ or $\mathrm{CD} 14^{\text {low }} \mathrm{PM} \varphi$ populations (Fig. 2D). Although hormone treatment did not alter the abundance of any of these immune cell populations (Fig. 2E and Supplementary Fig. 1), samples from patients on hormones were excluded from the subsequent analysis to avoid any confounding effects of this variable on other disease parameters.

\section{Peritoneal macrophages are not altered by infertility} or heavy menstrual bleeding

We next assessed whether the co-morbidities infertility or heavy menstrual bleeding (HMB), recorded as part of patient cohort data collection, could affect the abundance of $\operatorname{PM} \varphi$ subpopulations. Infertility did not alter the abundance of CD14 high or CD14low macrophages in women with endometriosis (Fig. $2 \mathrm{~F}$ ). $\mathrm{PM} \varphi$ populations were not altered by the presence of HMB (Fig. 2G).

\section{CD14high peritoneal macrophages are increased in} women with endometriosis

$\operatorname{PM} \varphi$ were assessed in all women within the cohort who had regular cycles and were not receiving hormone treatment and comparisons made between 'No endo' and those with endometriosis (pooled 'rASRM I-IV'). CD14high $\operatorname{PM} \varphi$ were significantly higher in women with endometriosis (rASRM I-IV) compared to women without disease (Fig. 3A, $P<0.05$ ). No significant difference was found for $\operatorname{CD} 14^{\text {low }} \operatorname{PM} \varphi$, although the average appeared lower for women with endometriosis (Fig. 3B). To assess the relationship between the two populations we calculated the ratio of CD14 $4^{\text {high }}$ to $\mathrm{CD} 14^{\text {low }}$ macrophages. $\mathrm{CD} 14^{\text {high }} \mathrm{PM} \varphi$ were the predominant population in the peritoneal cavity and this difference was more pronounced in women with endometriosis (Fig. 3C) and with increasing rASRM stage (Fig. 3D). Analysis according to rASRM stage (Fig. 3C) identified a significant difference between subpopulations of CD14 $4^{\text {high }}$ and $\mathrm{CD} 14^{\text {low }} \mathrm{PM} \varphi$ in women classified as rASRM stage I $(P<0.01)$, II $(P<0.0001)$ or III/IV $(P<0.001)$. No significant difference was found between $\mathrm{CD} 14^{\text {high }}$ and $\mathrm{CD} 14^{\text {low }} \mathrm{PM} \varphi$ in women without endometriosis.

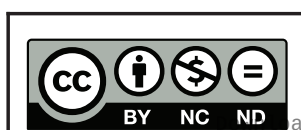

This work is licensed under a Creative Commons Attribution-NonCommercial-NoDerivatives 4.0 International License. 
A

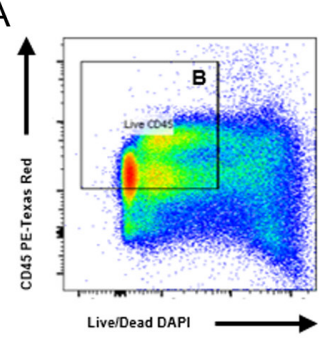

D

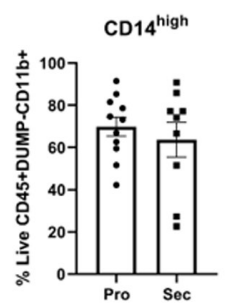

$\mathrm{F}$

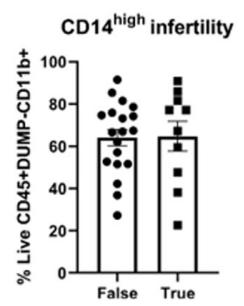

B

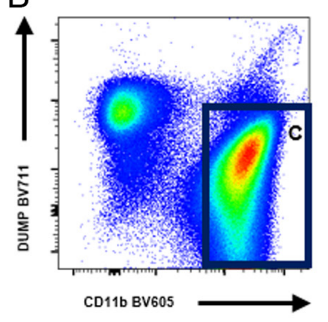

E

C

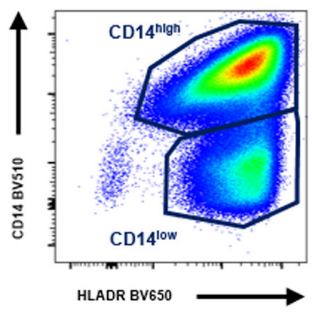

CD $14^{\text {high }}$
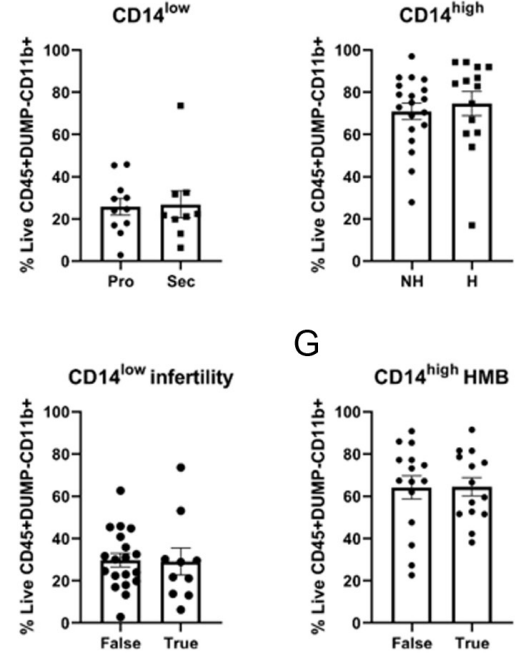

G

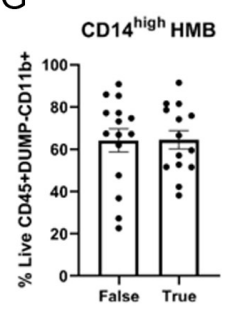

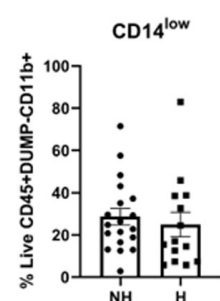

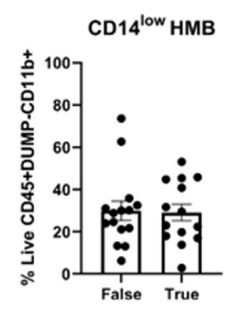

Figure $\mathbf{2}$ Analysis of peritoneal fluid immune cells from women with diagnosed endometriosis reveals two populations of peritoneal macrophages that are not altered by hormones or other reproductive health disorders.

Representative flow cytometry plots from pelvic peritoneal fluid (PF) immune cells. Cells were gated to select; (A) Live, CD45 (all immune cells) and (B) CD11 b+ (myeloid cells), CD3-CD19-CD56('DUMP'; T, B and NK cells). (C) Two peritoneal macrophage $(\mathrm{PM} \varphi)$ subpopulations were identified that were HLA-DR ${ }^{+}$and $\mathrm{CD} 14^{+}$(High vs low). (D) Phase of the menstrual cycle (Pro: proliferative; Sec: secretory) did not affect the abundance of CD14high ( $t$ test, $P=0.5035$ ) or CD14low cells (Mann-Whitney test, $P=0.7664$ ) thus samples were thereafter not stratified on this basis. (E) The abundance of CD14high (MannWhitney test, $P=0.3723$ ) or CD14low cells (MannWhitney test, $P=0.3345$ ) did not differ if women were not on hormones $(\mathrm{NH})$ or on a hormonal treatment $(\mathrm{H})$. (F) The abundance of $\mathrm{CD} 14$ high or CD14low peritoneal macrophages (PM $\varphi$ ) did not differ between women reporting infertility (True) and those who did not (False). (G) The abundance of CD14high or CD14low PM $\varphi$ in women with endometriosis did not differ between women reporting HMB (True) and those who did not (False).

\section{Peritoneal macrophages correlate with pelvic pain scores in women with endometriosis and are influenced by pain subtype}

The abundance of either CD14high or CD14low $\mathrm{PM} \varphi$ was compared to pain scores in women with endometriosis who were not on hormones and Pearson correlation calculated. In women who were diagnosed with endometriosis (rASRM I-IV), CD14 ${ }^{\text {high }}$ PM $\varphi$ did not significantly correlate with pain score (Fig. 4, All, $P=0.057$ ), although a trend for negative correlation was evident. However, CD14low $\mathrm{PM} \varphi$ showed a moderate positive relationship with the pain score that was borderline significant (Fig. 4, All, $P=0.047)$. No correlation was observed in women without endometriosis, irrespective of hormone status, but these subgroups had the fewest patients (Supplementary Fig. 2).

To account for different pain subtypes within women with endometriosis, patients were stratified based on reported presence of pain subtypes; dysmenorrhea (DM), dyspareunia (DP) and non-menstrual pain (NMP). Dysmenorrhea was present in the majority of endometriosis patients (89.5\%, Supplementary Table 4) and comparisons were therefore similar to those that accounted for all endometriosis patients; CD14 ${ }^{\text {high }} \mathrm{PM} \varphi$ did not significantly correlate with pain score (Fig. 4, DM,
$P=0.057)$ but $\mathrm{CD} 14^{\text {low }} \mathrm{PM} \varphi$ showed a significant positive relationship with pain score (Fig. 4 , DM, $P=0.049$ ).

Dyspareunia was less common than dysmenorrhea in endometriosis patients (73.7\%, Supplementary Table 4). CD14 ${ }^{\text {high }}$ PM $\varphi$ had a significant negative correlation with pain score (Fig. 4, DP, $P=0.044$ ) and CD14low PM $\varphi$ had a significant positive correlation with pain score (Fig. 4, DP, $P=0.031)$.

Non-menstrual pain (NMP) was commonly reported in endometriosis patients (84.2\%, Supplementary Table 4). CD14 high $\mathrm{PM} \varphi$ had a significant negative correlation with pain score (Fig. 4, NMP, $P=0.015$ ) and CD14 low $\mathrm{PM} \varphi$ had a significant positive correlation with pain score (Fig. 4, NMP, $P=0.015$ ).

Based on calculated correlation coefficient values $(\mathrm{r})$, the strongest relationship between $\mathrm{PM} \varphi$ and pain scores was in women with endometriosis who reported experiencing pain outwith menses (non-menstrual pain; NMP). Notably, multiple pain subtypes were present in many patients and stratification was therefore based on the presence of a given subtype. To account for potential interactions between pain subtypes, correlations were also calculated on the basis of whether patients reported both DM and DP, DM and NMP, DP and NMP or all three

This work is licensed under a Creative Commons Attribution-NonCommercial-NoDerivatives 4.0 International License. ded from Bioscientifica.com at 04/26/2023 07:08:36AM 
A

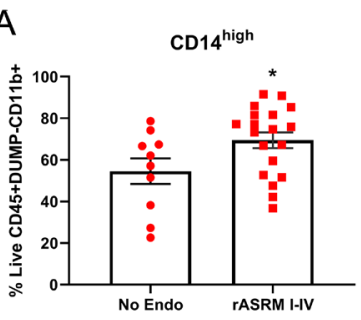

C

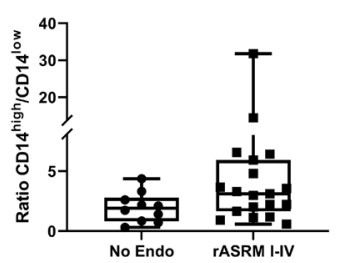

E

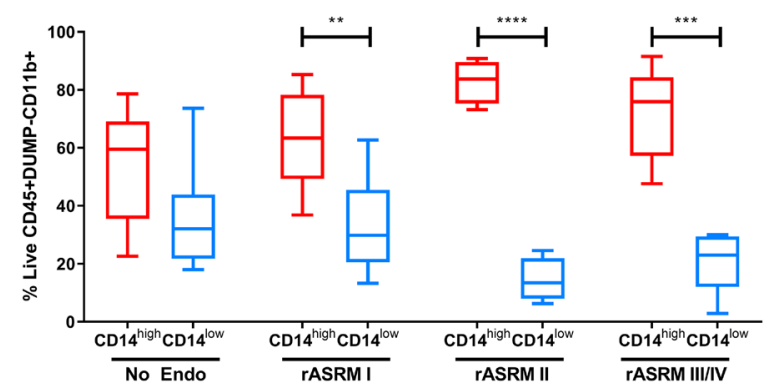

Figure 3 CD14 ${ }^{\text {high }}$ peritoneal macrophages are increased in women with endometriosis. (A) Frequency of CD14 high peritoneal macrophages (PM $\varphi$ ) was greater in women with endometriosis (rASRM stages I-IV, $n=19, t$ test, $P<0.05$ ) than those without endometriosis ('No endo'; $n=10$ ). (B) Frequency of $C D 14^{\text {low }} \mathrm{PM} \varphi$ was not significantly different in women with endometriosis compared to those without disease. (C) The ratio of CD14high to CD14low PM $\varphi$ was greater in women with endometriosis (rASRM I-IV) than those without endometriosis (no endo). (D) The ratio of CD14 high to CD14low PM $\varphi$ was greater in women from each rASRM stage than those without endometriosis (no endo). (E) CD14 high PM $\varphi$ were significantly higher than CD14low PM $\varphi$ in women with endometriosis stratified according to $\operatorname{rASRM~I~}(n=10, P<0.01)$, rASRM II $(n=4, P>$ $0.0001)$ and rASRM III/IV $(n=5, P>0.001)$ but not in women without endometriosis ( $n=9$; two-way ANOVA). ${ }^{\star} P<0.05$, $* * P<0.01, * \star \star P<0.001$, $\star \star \star \star * P<0.0001$.

subtypes but this did not affect the correlations reported by individual pain subtypes (Supplementary Table 4).

\section{Peritoneal macrophages correlate with severity of pelvic pain in women irrespective of endometriosis diagnosis}

To extend the data on women with endometriosis, the abundance of either CD14 $4^{\text {high }}$ or $\operatorname{CD} 14^{\text {low }} \operatorname{PM} \varphi$ was also compared to pain scores using data from all women in our cohort who were not on hormones (No endo and rASRM I-IV combined) and Pearson correlation calculated (Fig. 5). A significant correlation was found for all patients
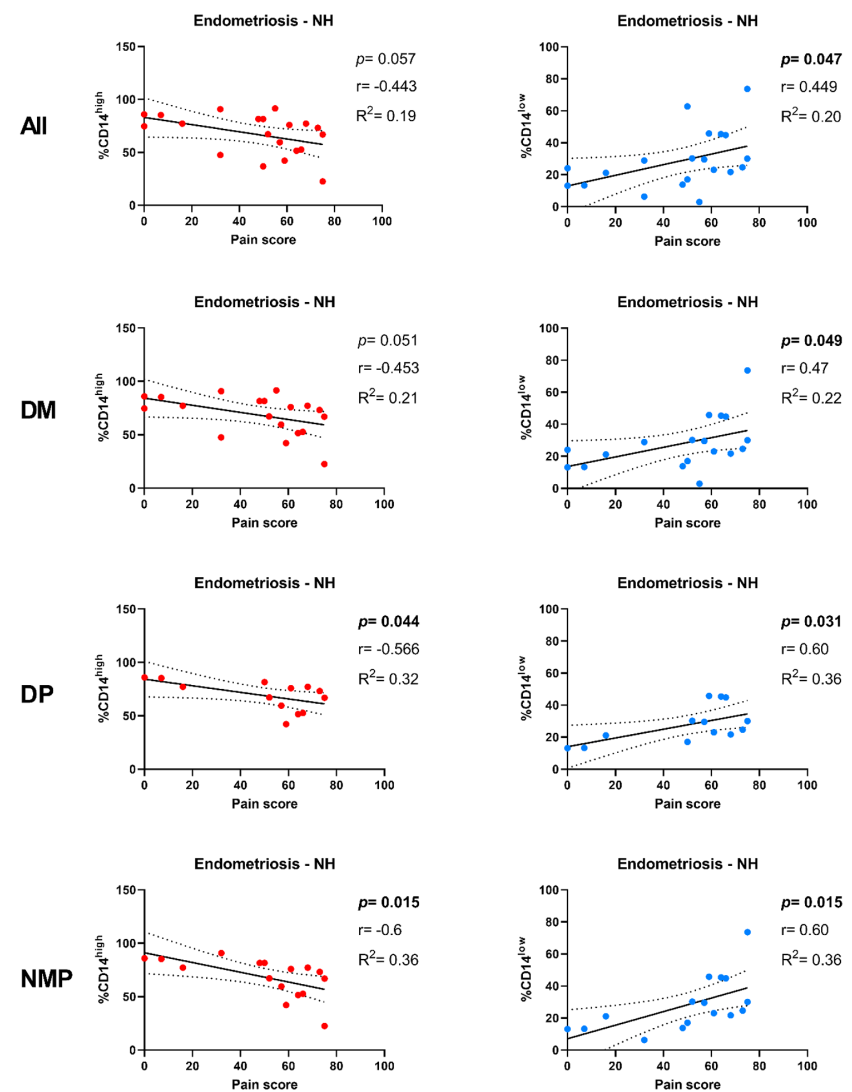

Figure 4 Peritoneal macrophages correlate with the severity of pelvic pain in women with endometriosis and are influenced by pain subtype. Frequency of $\mathrm{CD} 14^{\text {high }} \mathrm{PM} \varphi$ did not significantly correlate with pain scores ( $n=20, P=0.057$ ) but CD14low PM $\varphi$ showed a significant positive correlation with pain score in endometriosis patients ( $n=20, P=0.047$ ). Patients were stratified based on reported presence of pain subtypes; dysmenorrhea (DM), dyspareunia (DP) and non-menstrual pain (NMP). Endometriosis patients who reported dysmenorrhea showed moderate positive correlation between CD14low PM $\varphi$ and pain score $(n=19, P=$ 0.049 ) but no significant correlation between CD14high $\mathrm{PM} \varphi$ and pain score. Endometriosis patients who reported dyspareunia showed a significant negative correlation between CD14high $\mathrm{PM} \varphi$ and pain score $(n=$ $13, P=0.0441)$ and a significant positive correlation between CD14low PM $\varphi$ and pain score $(n=13, P=0.0301)$. Endometriosis patients who reported non-menstrual pain showed a significant negative correlation between CD14high PM $\varphi$ and pain score $(n=16, P=0.0147)$ and a significant positive correlation between $\mathrm{CD} 14^{\text {low }} \mathrm{PM} \varphi$ and pain score $(n=16, P=0.0146)$. $\mathrm{P}$ value, correlation coefficient $(r)$ and goodness of fit $\left(R^{2}\right)$ calculated by Pearson correlation. Line of best fit calculated by simple linear regression (black line) with 95\% Cls (dashed line). $\mathrm{NH}$, not on hormones. Attribution of pain subtype was not exclusive as multiple subtypes were reported in some patients.

for both CD14high (Fig. 5, All, $P=0.031$ ), and CD14 low $\mathrm{PM} \varphi$ (Fig. 5, All, $P=0.032$ ) with pain score. This was also true when data were stratified by pain subtype.

Dysmenorrhea was present in most patients $(80 \%$, Supplementary Table 4). In women with dysmenorrhea, $\mathrm{CD} 14^{\text {high }} \mathrm{PM} \varphi$ negatively correlated with pain score (Fig. 5, $\mathrm{DM}, P=0.021)$ and $\mathrm{CD} 14^{\text {low }} \mathrm{PM} \varphi$ showed a significant 

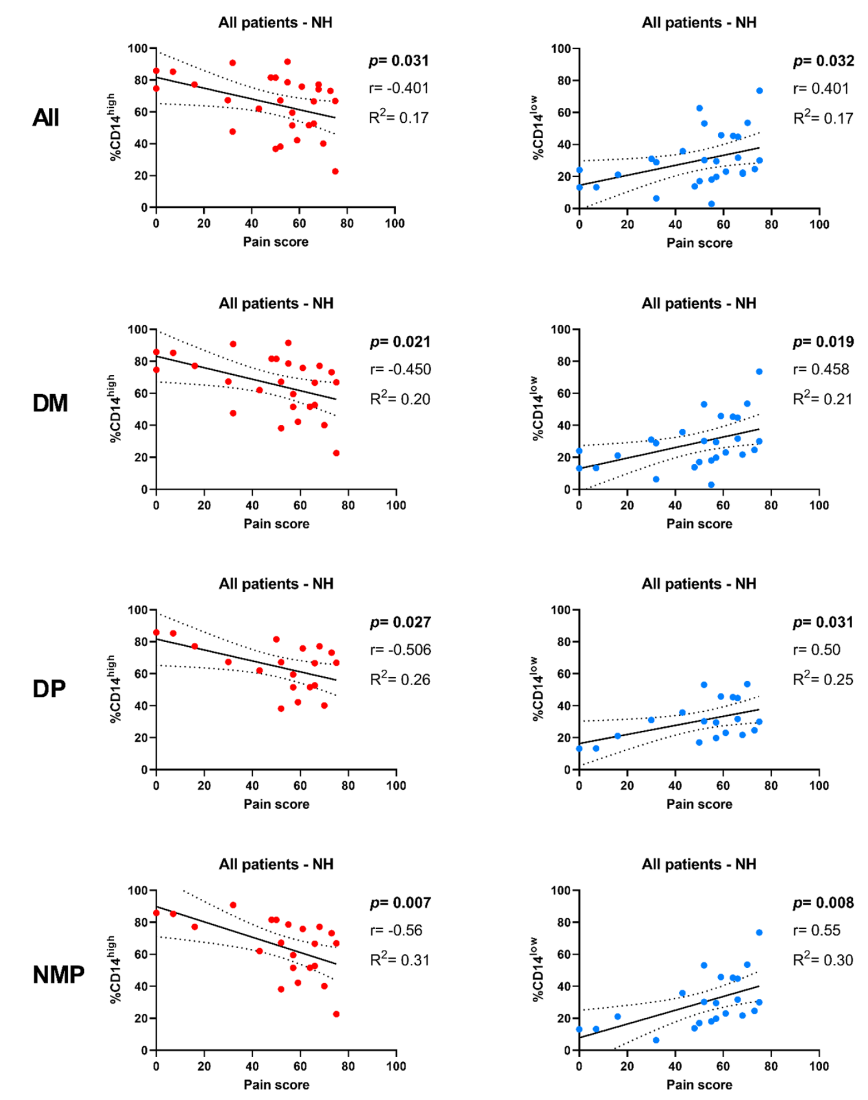

Figure $\mathbf{5}$ Peritoneal macrophages correlate with the severity of pelvic pain in women with suspected endometriosis irrespective of diagnosis. Frequency of $\mathrm{CD} 14^{\text {high }} \mathrm{PM} \varphi$ significantly negatively correlated with pain score $(n=28, P=0.031)$ and CD14low PM $\varphi$ showed a significant positive correlation with pain score $(n=28, P=0.032)$ in all patients irrespective of the endometriosis diagnosis. Patients were stratified based on reported presence of pain subtypes; dysmenorrhea (DM), dyspareunia (DP) and non-menstrual pain (NMP). Patients who reported dysmenorrhea showed a significant negative correlation between $C D 14^{\text {high }} \mathrm{PM} \varphi$ and pain score ( $n=26, P=0.0212$ ) and a significant positive correlation between CD14low PM $\varphi$ and pain score $(n=26, P=0.01865)$. Patients who reported dyspareunia showed a significant negative correlation between CD14high PM $\varphi$ and pain score $(n=19, P=0.0270)$ and a significant positive correlation between CD14low PM $\varphi$ and pain score $(n=19, P=0.0306)$. Patients who reported pain non-menstrual pain showed a significant negative correlation between CD14high $\mathrm{PM} \varphi$ and pain score $(n=22, P=$ 0.0074 ) and a significant positive correlation between CD14low PM $\varphi$ and pain score ( $n=22, P=0.0083$ ). P value, correlation coefficient $(r)$ and goodness of fit $\left(R^{2}\right)$ calculated by Pearson correlation. Line of best fit calculated by simple linear regression (black line) with 95\% confidence intervals (dashed line). NH, not on hormones. Attribution of pain subtype was not exclusive as multiple subtypes were reported in some patients.

positive relationship with pain score (Fig. 5, DM, $P=0.019$ ). Dyspareunia was less common than dysmenorrhea (70\%, Supplementary Table 4). CD14 high $\operatorname{PM} \varphi$ had a significant negative correlation with pain score (Fig. 5, DP, $P=0.027$ ) and $\mathrm{CD} 14^{\text {low }} \mathrm{PM} \varphi$ had a significant positive correlation with pain score (Fig. 5, DP, $P=0.031$ ). Non-menstrual pain (NMP) was commonly reported $(76.7 \%$, Supplementary
Table 4). CD14 high $\operatorname{PM} \varphi$ had a strong significant negative correlation with pain score (Fig. 5, NMP, $P=0.007$ ) and $\mathrm{CD} 14^{\text {low }} \mathrm{PM} \varphi$ had a significant positive correlation with pain score (Fig. 5, NMP, $P=0.008$ ). Similar to the analysis of data from the subgroup of women who were diagnosed with endometriosis, when all patients were considered the strongest relationship between $\operatorname{PM} \varphi$ and pain scores was in women who reported experiencing non-menstrual pain (CD14 high $\mathrm{r}=-0.56$; CD14 low, $\mathrm{r}=0.55)$.

\section{Discussion}

Peritoneal macrophages are believed to play a fundamental role in the pathophysiology of endometriosis (Bacci et al. 2009, Yuan et al. 2016, Horne \& Saunders 2019, Zondervan et al. 2020) but to date, information regarding different phenotypes of macrophage and how they relate to pain symptoms in women has been limited. In this study, we performed multi-parameter flow cytometry analysis of peritoneal fluid immune cells from women undergoing a diagnostic laparoscopy for suspected endometriosis. We believe our data are the first to demonstrate that a CD14 high $\operatorname{PM} \varphi$ subset are increased in women with laparoscopically diagnosed endometriosis and that the abundance of both $\mathrm{CD} 14^{\text {high }}$ and $\mathrm{CD} 14^{\text {low }} \mathrm{PM} \varphi$ populations correlate with pain symptoms independent of the endometriosis diagnosis.

In a previous study, researchers reported an increase in the abundance of macrophages in the peritoneal fluid of 37 women with endometriosis based on the expression of the macrophage marker CD68 (Beste et al. 2014) which is consistent with the subset specific increase in CD14 high detected in the current study. Based on relative abundance and comparative transcriptional profiling in other studies (Irvine et al. 2016), we believe CD14 $4^{\text {high }}$ and CD14 $4^{\text {low }}$ to be broadly equivalent to large (LpM) or small (SpM) peritoneal macrophage populations described in mice (Cassado et al. 2015). In response to peritoneal cavity inflammation in mice, LpM decrease and this is associated with infiltration of monocytes that replenish the LpM pool. Notably, it is not possible to distinguish between 'resident' $\mathrm{PM} \varphi$ and infiltrating monocytes in women as they both express CD14. Thus, the increase in CD14 high cells we detected in women with endometriosis may in part be accounted for by monocyte infiltration. This may also be reflected by the increase in the proportion of CD14 high relative to CD14 low we detected with rASRM stages I, II and III/IV which may be associated with increased peritoneal inflammation in response to disease burden. Future studies will need to 
profile paired monocyte and $\operatorname{PM} \varphi$ samples from women with endometriosis in order to distinguish their respective contribution to the altered peritoneal microenvironment that exists in women with endometriosis.

We assessed pain scores in 52 women who were scheduled to have a diagnostic laparoscopy for suspected endometriosis. Pelvic pain symptoms were recorded using the pain dimension of the EHP-30 questionnaire, an extensively validated endometriosis-specific healthrelated quality of life measurement (Jones et al. 2004, Bourdel et al. 2019). We found that pain scores in our cohort of women with endometriosis (mean and s.E.M., $45.5 \pm 5.54$ ) were comparable to previous data generated using the EHP-30 questionnaire in larger cohorts of women from the UK (mean pain score 43.3, $n=594$ women), USA (mean pain score $49.3, n=225$ women) and Australia (mean pain score $40.7, n=189$ women) (Jones et al. 2006, Jenkinson et al. 2008, Khong et al. 2010) so we believe they are representative of this patient group. These previous studies focused on pain scores in women with surgically diagnosed endometriosis but in the current study, we also analysed pain scores in women who did not receive a diagnosis of endometriosis at laparoscopy. Interestingly, mean pain score in the 'no endo' group was higher (mean and s.E.M., $57.3 \pm 4.78$ ) than women with endometriosis but variation within groups meant this was not statistically significant when compared to pain scores in women with endometriosis. Pain scores were also not significantly different between patients graded as rASRM stage I (47.3), II (38.3) or III/IV (47.8). Thus, in the current patient cohort, neither a diagnosis of endometriosis nor rASRM stage affected pain scores. Taken together, these data confirm previous findings that pain symptoms do not correlate well with disease presentation (Vercellini et al. 2007).

We profiled peritoneal cavity immune cells to determine if changes in the inflammatory environment could predict the severity of pain symptoms. Broadly, we found a negative correlation between $\mathrm{CD} 14^{\text {high }} \mathrm{PM} \varphi$ and pelvic pain and a positive correlation between $\mathrm{CD} 14^{\text {low }} \mathrm{PM} \varphi$ and pelvic pain. These correlations were significant in patients with endometriosis but also when the whole patient cohort was evaluated independently of the endometriosis diagnosis. The correlation between the abundance of peritoneal macrophage subsets and pain score was more pronounced when patients were stratified by pain subtype. Patients who reported nonmenstrual pain showed a marked significant correlation between peritoneal macrophages and pain score and these patients also showed the strongest correlation coefficient.
This suggests a potential disconnect between pain and menstrual dysfunction, reinforcing the possibility that inflammatory changes in the peritoneal environment may be a greater driver of pain symptoms in some patients. Thus, although some patients may have no 'active' or visible endometriosis at the time of laparoscopy they may exhibit detectable changes in macrophages which correlate to pelvic pain.

A previous study reported a correlation between concentrations of IL6 in peritoneal fluid and pelvic pain symptoms in women with endometriosis (Velasco et al. 2010). Notably, $\operatorname{PM} \varphi$ are reported to be the principle source of IL- 6 and this cytokine is increased in peritoneal fluid in women with endometriosis (Wu et al. 1999). Pain symptoms may therefore be affected by secretion of proinflammatory cytokines from $\mathrm{PM} \varphi$ in response to 'active' disease or as a legacy of previous inflammation from 'historic' disease. Thus, further profiling of peritoneal macrophages to account for both their phenotype and function could provide new insights into the relationship between endometriosis and pelvic pain symptoms. Notably, accounting for pain subtype as well as the severity of pelvic pain symptoms will provide the greatest resolution for identifying specific mediators with the potential to promote inflammatory pain.

Current treatment approaches for endometriosis focus on either surgical removal of disease or hormonal suppression of lesion growth but this does not improve pain symptoms in all women (Taylor et al. 2017, Saraswat et al. 2018, Pokrzywinski et al. 2020). Furthermore, symptomatic relapse or further surgery occurs in $24-72 \%$ of women within 7 years of the initial surgery (reviewed in Guo \& Martin 2019). We have previously shown that sham surgery in the peritoneal cavity of mice dramatically alters the composition of peritoneal macrophages by promoting increased replenishment by blood monocytes (Bain et al. 2020). Notably, this was detectable for up to 8 weeks post-surgery consistent with surgical intervention having a lasting effect on the peritoneal inflammatory environment in mice (Bain et al. 2020). In the context of the current results, we, therefore, speculate that surgical treatment itself could alter the peritoneal environment which raises the possibility that surgery could exacerbate pain symptoms in some patients. This may explain some of the contradictory findings assessing the impact of surgery on pain symptoms in women with superficial peritoneal endometriosis (Horne et al. 2019). The results of the current study suggest that assessing peritoneal macrophages could provide useful insights into the impact of surgical treatment as well as the inflammatory ( 
profile of patients with recurrent pain. Our new data also highlights the possibility that pain responses to nonsurgical treatments may be influenced by peritoneal macrophage function. For example, GnRH agonists which reduce pelvic pain in women with endometriosis (Brown et al. 2010) can increase PM $\varphi$ cytotoxicity (Braun et al. 1992) and reduce peritoneal fluid concentrations of the pain-correlated cytokine IL6 (Kuroda et al. 2010). GnRH modulators do not improve pain symptoms in all women (Taylor et al. 2017, Pokrzywinski et al. 2020) which could be accounted for by baseline differences in peritoneal macrophage phenotype or function. We, therefore, propose that profiling $\mathrm{PM} \varphi$ may have future utility for predicting response to surgical treatment and as a means for assessing the impact of medical treatments on inflammatory processes that drive pain symptoms.

\section{Conclusions}

The novel findings in this study provide new evidence for a link between the abundance of peritoneal macrophage subpopulations and the experience of pelvic pain symptoms in women. Collectively, our data suggest that the inflammatory profile of the peritoneal environment may be a better predictor of pain symptoms than the presence/volume of endometriosis lesions identified during laparoscopy. Reframing this perspective opens a path to future targeted therapies that aim to alter peritoneal macrophage function and reduce pelvic pain symptoms in women.

\section{Supplementary materials}

This is linked to the online version of the paper at https://doi.org/10.1530/ RAF-20-0072.

\section{Declaration of interest}

A W H has received honoraria for consultancy for Ferring, Roche, Nordic Pharma, and Abbvie. B D L is now an employee of Bayer AG Pharma, Preclinical Research. Andrew Horne is a Co-Editor-in-Chief of Reproduction and Fertility. Andrew Horne was not involved in the review or editorial process for this paper, on which he is listed as an author.

\section{Funding}

Flow cytometry data were generated with support from the QMRI Flow Cytometry and Cell Sorting Facility, University of Edinburgh. Studies were supported by MRC Programme Grants G1100356/1 to P T K S and MR/ N024524/1 to P T K S, D A G and A W H. Studentship for B D L was funded by a grant to the MRC Centre for Reproductive Health (G1002033). A W H has received honoraria for consultancy for Ferring, Roche, Nordic Pharma, and Abbvie.

\section{Author contribution statement}

Experimental design; D A G, A W H, P T K S, experimental procedures; D A G, B D, F C, manuscript preparation; D A G, F C, A W H, P T K S.

\section{Acknowledgements}

The authors thank Dr Alistair Williams for histological evaluations of endometrial tissue and members of the clinical research team Helen Dewart, Jennifer Rowan and Ashleigh Symington for patient recruitment and sample collection. The authors thank members of PTKS laboratory for technical support, particularly Arantza Esnal-Zufiaurre for histology preparations and Olympia Kelepouri for sample processing. The authors thank Dr Luca Cassetta and Dr Samanta Mariani for their advice in designing antibody panels for flow cytometry analysis.

\section{References}

1997 Revised American Society for Reproductive Medicine classification of endometriosis: 1996. Fertility and Sterility 67 817-821. (https://doi. org/10.1016/s0015-0282(97)81391-x)

Arici A, Oral E, Attar E, Tazuke SI \& Olive DL 1997 Monocyte chemotactic protein-1 concentration in peritoneal fluid of women with endometriosis and its modulation of expression in mesothelial cells. Fertility and Sterility 67 1065-1072. (https://doi.org/10.1016/ s0015-0282(97)81440-9)

Bacci M, Capobianco A, Monno A, Cottone L, Di Puppo F, Camisa B, Mariani M, Brignole C, Ponzoni M, Ferrari S, et al. 2009 Macrophages are alternatively activated in patients with endometriosis and required for growth and vascularization of lesions in a mouse model of disease. American Journal of Pathology $\mathbf{1 7 5}$ 547-556. (https://doi.org/10.2353/ajpath.2009.081011)

Bain CC, Gibson DA, Steers NJ, Boufea K, Louwe PA, Doherty C, González-Huici V, Gentek R, Magalhaes-Pinto M, Shaw T, et al. 2020 Rate of replenishment and microenvironment contribute to the sexually dimorphic phenotype and function of peritoneal macrophages. Science Immunology 5 eabc4466. (https://doi. org/10.1126/sciimmunol.abc4466)

Beste MT, Pfäffle-Doyle N, Prentice EA, Morris SN, Lauffenburger DA, Isaacson KB \& Griffith LG 2014 Molecular network analysis of endometriosis reveals a role for c-jun-regulated macrophage activation. Science Translational Medicine 6 222ra16. (https://doi.org/10.1126/scitranslmed.3007988)

Bourdel N, Chauvet P, Billone V, Douridas G, Fauconnier A, Gerbaud L \& Canis M 2019 Systematic review of quality of life measures in patients with endometriosis. PLOS ONE 14 e0208464. (https://doi.org/10.1371/journal.pone.0208464)

Braun DP, Gebel H, Rotman C, Rana N \& Dmowski WP 1992 The development of cytotoxicity in peritoneal macrophages from women with endometriosis. Fertility and Sterility 57 1203-1210. (https://doi. org/10.1016/S0015-0282(16)55074-2)

Brown J, Pan A \& Hart RJ 2010 Gonadotrophin-releasing hormone analogues for pain associated with endometriosis. Cochrane Database of Systematic Reviews Article Number: CD008475. (https://doi. org/10.1002/14651858.CD008475.pub2)

Budrys NM, Nair HB, Liu YG, Kirma NB, Binkley PA, Kumar S, Schenken RS \& Tekmal RR 2012 Increased expression of

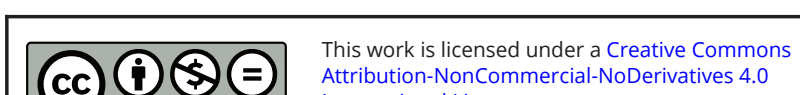

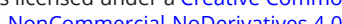
International License. ded from Bioscientifica.com at 04/26/2023 07:08:36AM 
macrophage colony-stimulating factor and its receptor in patients with endometriosis. Fertility and Sterility 97 1129.e1-1135.e1. (https://doi.org/10.1016/j.fertnstert.2012.02.007)

Capobianco A, Monno A, Cottone L, Venneri MA, Biziato D, Di Puppo F, Ferrari S, De Palma M, Manfredi AA \& RovereQuerini P 2011 Proangiogenic Tie2(+) macrophages infiltrate human and murine endometriotic lesions and dictate their growth in a mouse model of the disease. American Journal of Pathology 179 2651-2659. (https://doi.org/10.1016/j.ajpath.2011.07.029)

Cassado Ados A, D'império Lima MR \& Bortoluci KR 2015 Revisiting mouse peritoneal macrophages: heterogeneity, development, and function. Frontiers in Immunology 6 225. (https:// doi.org/10.3389/fimmu.2015.00225)

Chuang PC, Wu MH, Shoji Y \& Tsai SJ 2009 Downregulation of CD36 results in reduced phagocytic ability of peritoneal macrophages of women with endometriosis. Journal of Pathology 219 232-241. (https://doi.org/10.1002/path.2588)

Crosignani P, Olive D, Bergqvist A \& Luciano A 2006 Advances in the management of endometriosis: an update for clinicians. Human Reproduction Update 12 179-189. (https://doi.org/10.1093/humupd/ dmi049)

Fauconnier A \& Chapron C 2005 Endometriosis and pelvic pain: epidemiological evidence of the relationship and implications. Human Reproduction Update 11 595-606. (https://doi.org/10.1093/ humupd/dmi029)

Forster R, Sarginson A, Velichkova A, Hogg C, Dorning A, Horne AW, Saunders PTK \& Greaves E 2019 Macrophagederived insulin-like growth factor-1 is a key neurotrophic and nerve-sensitizing factor in pain associated with endometriosis. FASEB Journal 33 11210-11222. (https://doi.org/10.1096/fj.201900797R)

Gogacz M, Gałczyński K, Wojtaś M, Winkler I, Adamiak A, Romanek-Piva K, Rechberger T \& Kotarski J 2017 Fas-related apoptosis of peritoneal fluid macrophages in endometriosis patients: understanding the disease. Journal of Immunology Research $\mathbf{2 0 1 7}$ 3175394. (https://doi.org/10.1155/2017/3175394)

Guo SW \& Martin DC 2019 The perioperative period: a critical yet neglected time window for reducing the recurrence risk of endometriosis? Human Reproduction 34 1858-1865. (https://doi. org/10.1093/humrep/dez187)

Hill JA, Faris HMP, Schiff I \& Anderson DJ 1988 Characterization of leukocyte subpopulations in the peritoneal fluid of women with endometriosis. Fertility and Sterility 50 216-222. (https://doi. org/10.1016/S0015-0282(16)60062-6)

Horne AW \& Saunders PTK 2019 SnapShot: endometriosis. Cell 179 1677.e1-1677.e1. (https://doi.org/10.1016/j.cell.2019.11.033)

Horne AW, Daniels J, Hummelshoj L, Cox E \& Cooper KG 2019 Surgical removal of superficial peritoneal endometriosis for managing women with chronic pelvic pain: time for a rethink? BJOG 126 1414-1416. (https://doi.org/10.1111/1471-0528.15894)

Irvine KM, Banh X, Gadd VL, Wojcik KK, Ariffin JK, Jose S, Lukowski S, Baillie GJ, Sweet MJ \& Powell EE 2016 CRIgexpressing peritoneal macrophages are associated with disease severity in patients with cirrhosis and ascites. JCI Insight $\mathbf{1}$ e86914. (https://doi.org/10.1172/jci.insight.86914)

Jamieson DJ \& Steege JF 1996 The prevalence of dysmenorrhea, dyspareunia, pelvic pain, and irritable bowel syndrome in primary care practices. Obstetrics and Gynecology 87 55-58. (https://doi. org/10.1016/0029-7844(95)00360-6)

Jenkinson C, Kennedy S \& Jones G 2008 Evaluation of the American version of the 30-item Endometriosis Health Profile (EHP-30). Quality of Life Research 17 1147-1152. (https://doi.org/10.1007/s11136-0089403-9)

Jones G, Kennedy S, Barnard A, Wong J \& Jenkinson C 2001 Development of an endometriosis quality-of-life instrument: the Endometriosis Health Profile-30. Obstetrics and Gynecology 98 258-264. (https://doi.org/10.1016/s0029-7844(01)01433-8)
Jones G, Jenkinson C \& Kennedy S 2004 Evaluating the responsiveness of the endometriosis health profile questionnaire: the EHP-30. Quality of Life Research 13 705-713. (https://doi.org/10.1023/ B:QURE.0000021316.79349.af)

Jones G, Jenkinson C, Taylor N, Mills A \& Kennedy S 2006 Measuring quality of life in women with endometriosis: tests of data quality, score reliability, response rate and scaling assumptions of the Endometriosis Health Profile Questionnaire. Human Reproduction 21 2686-2693. (https://doi.org/10.1093/humrep/ del231)

Kajitani T, Maruyama T, Asada H, Uchida H, Oda H, Uchida S, Miyazaki K, Arase T, Ono M \& Yoshimura Y 2013 Possible involvement of nerve growth factor in dysmenorrhea and dyspareunia associated with endometriosis. Endocrine Journal 60 1155-1164. (https://doi.org/10.1507/endocrj.ej13-0027)

Khong SY, Lam A \& Luscombe G 2010 Is the 30-item Endometriosis Health Profile (EHP-30) suitable as a self-report health status instrument for clinical trials? Fertility and Sterility 94 1928-1932. (https://doi.org/10.1016/j.fertnstert.2010.01.047)

Kubicka U, Olszewski WL, Tarnowski W, Bielecki K, Ziółkowska A \& Wierzbicki Z 1996 Normal human immune peritoneal cells: subpopulations and functional characteristics. Scandinavian Journal of Immunology 44 157-163. (https://doi. org/10.1046/j.1365-3083.1996.d01-297.x)

Kuroda K, Kitade M, Kikuchi I, Kumakiri J, Matsuoka S, Kuroda M \& Takeda S 2010 Peritoneal vascular density assessment using narrow-band imaging and vascular analysis software, and cytokine analysis in women with and without endometriosis. Journal of Minimally Invasive Gynecology 17 21-25. (https://doi.org/10.1016/j. jmig.2009.09.003)

McKinnon KM 2018 Flow cytometry: an overview. Current Protocols in Immunology 120 5.1.1-5.1.11. (https://doi.org/10.1002/cpim.40)

Pokrzywinski RM, Soliman AM, Chen J, Snabes MC, Coyne KS, Surrey ES \& Taylor HS 2020 Achieving clinically meaningful response in endometriosis pain symptoms is associated with improvements in health-related quality of life and work productivity: analysis of 2 phase III clinical trials. American Journal of Obstetrics and Gynecology 222 592.e1-592.e10. (https://doi.org/10.1016/j. ajog.2019.11.1255)

Punnonen J, Teisala K, Ranta H, Bennett B \& Punnonen R 1996 Increased levels of interleukin- 6 and interleukin-10 in the peritoneal fluid of patients with endometriosis. American Journal of Obstetrics and Gynecology 174 1522-1526. (https://doi.org/10.1016/s00029378(96)70600-2)

Richter ON, Dorn C, Rösing B, Flaskamp C \& Ulrich U 2005 Tumor necrosis factor alpha secretion by peritoneal macrophages in patients with endometriosis. Archives of Gynecology and Obstetrics 271 143-147. (https://doi.org/10.1007/s00404-003-0591-9)

Ruiz-Alcaraz AJ, Tapia-Abellán A, Fernández-Fernández MD, Tristán-Manzano M, Hernández-Caselles T, SánchezVelasco E, Miras-López M, Martínez-Esparza M \& GarcíaPeñarrubia P 2016 A novel CD14high CD16high subset of peritoneal macrophages from cirrhotic patients is associated to an increased response to LPS. Molecular Immunology 72 28-36. (https:// doi.org/10.1016/j.molimm.2016.02.012)

Ruiz-Alcaraz AJ, Carmona-Martínez V, Tristán-Manzano M, Machado-Linde F, Sánchez-Ferrer ML, García-Peñarrubia P \& Martínez-Esparza M 2018 Characterization of human peritoneal monocyte/macrophage subsets in homeostasis: phenotype, GATA6, phagocytic/oxidative activities and cytokines expression. Scientific Reports 8 12794. (https://doi.org/10.1038/ s41598-018-30787-x)

Saraswat L, Ayansina D, Cooper KG, Bhattacharya S, Horne AW \& Bhattacharya S 2018 Impact of endometriosis on risk of further gynaecological surgery and cancer: a national cohort study. BJOG 125 64-72. (https://doi.org/10.1111/1471-0528.14793)

This work is licensed under a Creative Commons Attribution-NonCommercial-NoDerivatives 4.0 International License. ded from Bioscientifica.com at 04/26/2023 07:08:36AM 
Taylor HS, Giudice LC, Lessey BA, Abrao MS, Kotarski J, Archer DF, Diamond MP, Surrey E, Johnson NP, Watts NB, et al. 2017 Treatment of endometriosis-associated pain with elagolix, an Oral GnRH antagonist. New England Journal of Medicine 377 28-40. (https://doi.org/10.1056/NEJMoa1700089)

Tran LV, Tokushige N, Berbic M, Markham R \& Fraser IS 2009 Macrophages and nerve fibres in peritoneal endometriosis. Human Reproduction 24 835-841. (https://doi.org/10.1093/humrep/den483)

Velasco I, Acién P, Campos A, Acién MI \& Ruiz-Maciá E 2010 Interleukin- 6 and other soluble factors in peritoneal fluid and endometriomas and their relation to pain and aromatase expression. Journal of Reproductive Immunology 84 199-205. (https://doi. org/10.1016/j.jri.2009.11.004)

Vercellini P, Fedele L, Aimi G, Pietropaolo G, Consonni D \& Crosignani PG 2007 Association between endometriosis stage, lesion type, patient characteristics and severity of pelvic pain symptoms: a multivariate analysis of over 1000 patients. Human Reproduction 22 266-271. (https://doi.org/10.1093/humrep/del339)
Wu MY, Ho HN, Chen SU, Chao KH, Chen CD \& Yang YS 1999 Increase in the production of interleukin-6, interleukin-10, and interleukin- 12 by lipopolysaccharide-stimulated peritoneal macrophages from women with endometriosis. American Journal of Reproductive Immunology 41 106-111. (https://doi. org/10.1111/j.1600-0897.1999.tb00082.x)

Yuan M, Li D, AN, Li M, Zhang Q, L \& Wang G 2016 Rediscovering peritoneal macrophages in a murine endometriosis model. Human Reproduction 32 94-102. (https://doi.org/10.1093/humrep/dew274)

Zondervan KT, Becker CM \& Missmer SA 2020 Endometriosis. New England Journal of Medicine 382 1244-1256. (https://doi.org/10.1056/ NEJMra1810764)

Received in final form 3 February 2021

Accepted 1 March 2021

Accepted Manuscript published online 1 March 2021 (c) 2021 The authors Published by Bioscientifica Ltd
This work is licensed under a Creative Commons Attribution-NonCommercial-NoDerivatives 4.0 International License. ded from Bioscientifica.com at 04/26/2023 07:08:36AM 